\title{
Comparison of the Icare ic100 Rebound Tonometer and the Goldmann Applanation Tonometer in 1,000 Eyes
}

\author{
Arjun Gopal Subramaniam ${ }^{a, b}$ Penelope Allen ${ }^{a, c}$ Tze'Yo Toh $^{a}$ \\ ${ }^{a}$ Tasmanian Eye Institute, Launceston, TAS, Australia; 'baunceston Clinical School, College of Health and Medicine,

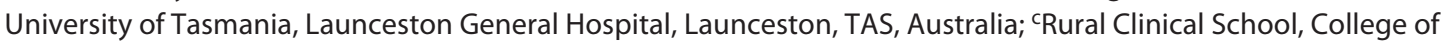 \\ Health and Medicine, University of Tasmania, Hospitals' Campus, Burnie, TAS, Australia
}

\section{Keywords}

Intraocular pressure - Icare rebound tonometer · Goldmann applanation tonometer

\begin{abstract}
Background: Measurement of intraocular pressure (IOP) forms a crucial component in the diagnosis and management of glaucoma. Many devices have been developed to measure IOP with Goldmann applanation tonometry (GAT) considered the gold standard for IOP measurement. Objectives: The objective was to compare the IOP measured using Icare ic100 and GAT. Methods: This cross-sectional study measured IOP in 1,000 eyes (500 left and 500 right) using the Icare ic100 and GAT. Central corneal thickness (CCT) was measured using a hand-held pachymeter. IOP measurements were investigated in all eyes, by IOP substrata, by CCT group, by number of topical glaucoma medications, and diagnosis. Results: There was moderate agreement between ic100 and GAT IOP measurements (intraclass correlation coefficient 0.73 ). Mean IOP was significantly lower when measured by ic100 than by GAT ( $12.1 \mathrm{vs.} 16.2 \mathrm{~mm} \mathrm{Hg}, p<0.0001$ ). Mean ic100 IOPs were also significantly lower than mean GAT IOPs within each IOP strata $\leq 12$ (7.9 vs. $9.7 \mathrm{~mm} \mathrm{Hg}, p<$ 0.0001 ), $13-21$ (12.1 vs. $16.6 \mathrm{~mm} \mathrm{Hg}, p<0.0001$ ), and $\geq 22$
\end{abstract}

(18.4 vs. $25.2 \mathrm{~mm} \mathrm{Hg}, p<0.0001$ ) and within each subanalysis. Conclusions: The Icare tonometer consistently under estimated IOP compared to GAT, irrespective of CCT ranges and other subgroup analyses. The mean difference of 4.2 $\mathrm{mm} \mathrm{Hg}$ can have significant clinical implications, particularly in the management of glaucoma patients.

(C) 2020 S. Karger AG, Basel

\section{Introduction}

Measurement of intraocular pressure (IOP) forms a crucial component in the diagnosis and management of multiple ocular conditions, especially glaucoma. IOP was first measured in 1865, when Von Graefe developed the first tonometry instrument [1]. Since then, many devices have been developed to measure IOP, including the Maklakoff tonometer, the Goldmann tonometer, the Perkins tonometer, rebound tonometers (RTs), and noncontact tonometers. Among these, Goldmann applanation tonometry (GAT) is still considered the gold standard for IOP measurement.

A number of studies have compared the accuracy of different tonometers, in particular between the GAT and versions of the Icare RT such as the Icare TAOi and the karger@karger.com

www.karger.com/ore

(C) 2020 S. Karger AG, Base

Karger ${ }^{\prime}=$
Penelope Allen

Rural Clinical School, University of Tasmania, Hospitals' Campus Brickport Road

Burnie, TAS 7320 (Australia)

penny.allen@utas.edu.au 
Icare Pro. Results of these studies have been inconsistent. Some studies reported undermeasurement of IOP by the Icare in comparison to GAT $[2,3]$. Other studies found Icare overestimates IOPs when compared to GAT [4-6]. Studies have also reported no significant differences in mean IOP measured by Icare compared to GAT [7-9].

Few studies have been published comparing the performance of Icare ic 100 tonometer against GAT [10-12]. As Icare rebound tonometry is widely used as a screening tool in IOP measurement, we set out to examine this method compared to GAT. The study's aim was to compare, in a clinical audit of 1,000 eyes, the IOP measured by Icare ic100 RT with the IOP measured by GAT.

\section{Materials and Methods}

The University of Tasmania Human Research Ethics Committee provided ethical approval for the research (reference: H18061). The study was performed according to the World Medical Association Declaration of Helsinki, at 2 ophthalmology centers in Tasmania, Australia.

The inclusion criterion was consecutive patients who attended routine clinical appointments during the study period. Exclusion criteria were measurements missing or significant corneal pathologies that may render difficult or inaccurate GAT.

The Icare device is a portable, easy-to-use RT that does not require the use of topical anesthesia. The ic100 used in our study has a positioning sensor and an automatic measurement mode unlike the Icare TAOi. It measures the IOP by striking the central cornea with a single use probe on the device tip [13]. An average of 6 measurements obtained by the device is shown as the IOP value [14].

Patients were asked to look at a distance and fixate on an object, while the Icare probe was held at a distance of 4-8 $\mathrm{mm}$, perpendicular to the central cornea. The Icare measurements were taken by a technician prior to the doctor who then carried out the GAT measurements after instilling a drop of proxymetacaine hydrochloride (5\%). The aim was to obtain 1 GAT measurement for each eye in order to minimize patients' discomfort. Icare and GAT measurements were then subsequently recorded onto a spreadsheet.

GAT works by the principle of applanation, where the corneal surface is applanated to a specified area of $3.06 \mathrm{~mm}$ at which the tear film surface tension and corneal rigidity are equal. The IOP (in $\mathrm{mm} \mathrm{Hg}$ ) is then measured indirectly by converting the force (in grams) required by the tonometer to achieve such area of applanation, by a factor of $10[13,14]$. The Icare device used in our study was calibrated daily and GAT was calibrated at the start of each consulting session by dialing the tonometer to " 0 " $\mathrm{mm} \mathrm{Hg}$ and observing movement of the tonometer prism.

Age, sex, central corneal thickness (CCT), diagnoses at presentation, and number of glaucoma medications were extracted from the electronic records. The data were imported into SPSS (version 26) (IBM Corporation 1989, 2019) for analysis. To determine concordance between Icare and GAT measures, mean IOP and standard deviations (SDs) measured by Icare and GAT were investigated. Mean IOP stratified by IOP measured with GAT (IOP: $\leq 12$, $13-21, \geq 22$ ) was investigated in left eyes, right eyes, and both eyes.
Mean IOP was also investigated stratified by age, CCT $(\leq 540$, $\geq 541$ ), and number of glaucoma medications $(0, \geq 1)$ for left and right eyes and by diagnosis (POAG, PACG, and PXG versus other diagnosis) in left and right eyes. Intraclass correlation coefficients (ICCs) with 95\% confidence intervals were calculated based on average absolute agreement using two-way mixed effects models. ICCs of 0.5 indicate poor reliability, $0.5-0.75$ indicates moderate reliability, $0.75-0.90$ indicates good reliability, and $>0.90$ excellent reliability. Paired $t$-tests were used to test for statistically significant differences in normally distributed data and Wilcoxon matched pairs signed-rank tests for nonparametric data. A scatterplot was produced for IOP measured by GAT and Icare for all eyes, left eyes, and right eyes. The method of least squares regression was used to determine the slopes, intercepts, and standard deviations (SD) of the linear regression lines. The Bland-Altman method (Bland and Altman, 1986, 1995) was used with the mean difference between the readings and the SD of the difference plotted. Tests were accepted as significant at $p<0.05$.

\section{Results}

There were 1,000 eyes among 500 patients (274 women and 226 men) included in the study. Mean age was 71 years (SD 10.8, range 24-97) and 412 patients (82.4\%) had a diagnosis of POAG, PACG, or PXG in 1 or both eyes.

Mean IOP measured by Icare was $12.1 \mathrm{~mm} \mathrm{Hg}$ (SD 4.6) and $16.2 \mathrm{~mm} \mathrm{Hg}$ (SD 5.3) measured by GAT, as shown in Table 1. The mean difference of -4.2 (3.4) was significant $\left(t_{(999)}=-39.1, p<0.0001\right)$. The ICC for Icare and GAT for all eyes was 0.73 , indicating moderate agreement between Icare and GAT. However, the 95\% confidence interval was wide $(95 \% \mathrm{CI}-0.90,0.89)$. There were also significant differences in IOP measured by Icare versus GAT in each of the IOP stratification groups. Intraclass correlation coefficients ranged from poor $(0.32,95 \% \mathrm{CI}-0.19,0.62)$ for left eye IOP in the 13-21 stratification group to moderate $(0.74,95 \%$ CI $-0.03,0.90)$ for all right eyes, with wide $95 \%$ confidence intervals for each subgroup ICC.

As shown in Table 2, bivariate analyses found mean Icare IOPs were significantly lower than mean GAT IOPs within both age subgroups, CCT subgroups, medication subgroups, and within diagnosis subgroups (POAG, PACG, and PX vs. other diagnosis). ICCs for the subgroups ranged from $0.58(95 \% \mathrm{CI}-0.22,0.84)$ to 0.81 (95\% CI 0.17, 0.93) with wide confidence intervals.

Figure 1 shows the scatterplot, linear regression line, and $95 \%$ confidence interval for GAT ( $y$-axis) and Icare ( $x$-axis) for all eyes. The Bland-Altman graph in Figure 2 shows the distribution of the difference in IOP $(y$ axis) and the means for IOP ( $x$-axis), between Icare and GAT measurements for all 1,000 eyes. There were 801 
Table 1. Mean IOP measurements and mean differences in IOP (Icare-GAT) and stratified by IOP measured with GAT (IOP: $\leq 12,13-$ $21, \geq 22$ )

\begin{tabular}{|c|c|c|c|c|c|}
\hline IOP group & $\begin{array}{l}\text { Icare, mean (SD), } \\
\text { mm Hg }\end{array}$ & $\begin{array}{l}\text { GAT, mean (SD), } \\
\text { mm Hg }\end{array}$ & ICC (95\% CI) & $\begin{array}{l}\text { Mean } \\
\text { difference (SD) }\end{array}$ & $p$ value \\
\hline \multicolumn{6}{|l|}{ Left eyes } \\
\hline $\mathrm{IOP} \leq 12^{\dagger}(n=110)$ & $8.0(2.7)$ & $10.0(2.3)$ & $0.53(0.08,0.73)$ & $-2.0(2.6)$ & $<0.001$ \\
\hline IOP $13-21^{\dagger}(n=320)$ & $12.1(3.1)$ & $16.6(2.2)$ & $0.32(-0.19,0.62)$ & $-4.5(2.8)$ & $<0.001$ \\
\hline $\mathrm{IOP} \geq 22^{\dagger}(n=70)$ & $18.5(4.4)$ & $25.2(3.4)$ & $0.37(-0.20,0.71)$ & $-6.7(3.7)$ & $<0.001$ \\
\hline All right $(n=500)$ & $12.1(4.8)$ & $16.1(5.6)$ & $0.74(-0.03,0.90)$ & $-4.1(3.5)$ & $<0.001$ \\
\hline $\mathrm{IOP} \leq 12^{\dagger}(n=126)$ & $7.8(3.2)$ & $9.4(2.5)$ & $0.65(0.36,0.79)$ & $-1.6(2.7)$ & $<0.001$ \\
\hline IOP $13-21^{\dagger}(n=295)$ & $12.2(3.1)$ & $16.6(2.3)$ & $0.34(-0.19,0.64)$ & $-4.4(2.9)$ & $<0.001$ \\
\hline $\mathrm{IOP} \geq 22^{\dagger}(n=79)$ & $18.4(4.8)$ & $25.2(3.5)$ & $0.34(-0.20,0.66)$ & $-6.8(4.3)$ & $<0.001$ \\
\hline \multicolumn{6}{|l|}{ Both eyes } \\
\hline
\end{tabular}

Paired $t$ tests used to investigate differences except where indicated by ${ }^{\dagger}$ where Wilcoxon signed-rank tests were used. ICC, intraclass correlation coefficient; IOP, intraocular pressure; GAT, Goldmann applanation tonometry; SD, standard deviation.

Table 2. Mean differences in IOP (Icare - GAT) stratified by CCT ( $\leq 540, \geq 541)$, number of glaucoma medications $(0, \geq 1)$, and diagnosis

\begin{tabular}{|c|c|c|c|c|c|}
\hline Group & $\begin{array}{l}\text { Icare, mean (SD), } \\
\text { mm Hg }\end{array}$ & $\begin{array}{l}\text { GAT, mean (SD), } \\
\text { mm Hg }\end{array}$ & ICC (95\% CI) & $\begin{array}{l}\text { Mean } \\
\text { difference (SD) }\end{array}$ & $p$ value \\
\hline \multicolumn{6}{|l|}{ Age group $(n=1,000)$} \\
\hline$\leq 71$ years $(n=470)$ & $13.2(4.6)$ & $17.3(5.3)$ & $0.70(-0.01,0.87)$ & $-4.1(3.8)$ & $<0.001$ \\
\hline$\geq 72$ years $(n=530)$ & $11.0(4.4)$ & $15.3(5.2)$ & $0.73(-0.17,0.90)$ & $-4.3(3.0)$ & $<0.001$ \\
\hline \multicolumn{6}{|l|}{$\operatorname{CCT}(n=1,000)$} \\
\hline$\leq 540(n=457)$ & $11.0(4.4)$ & $15.3(5.4)$ & $0.72(-0.13,0.89)$ & $-4.3(3.3)$ & $<0.001$ \\
\hline$\geq 541(n=543)$ & $13.0(4.6)$ & $17.0(5.1)$ & $0.72(-0.07,0.89)$ & $-4.1(3.4)$ & $<0.001$ \\
\hline \multicolumn{6}{|l|}{ Medications } \\
\hline \multicolumn{6}{|l|}{ Left $(n=500)$} \\
\hline $0(n=258)$ & $12.3(5.0)$ & $16.2(5.9)$ & $0.78(0.02,0.92)$ & $-3.9(3.4)$ & $<0.001$ \\
\hline$\geq 1(n=242)$ & $11.8(3.9)$ & $16.5(4.0)$ & $0.58(-0.22,0.84)$ & $-4.7(3.1)$ & $<0.001$ \\
\hline \multicolumn{6}{|l|}{ Right $(n=500)$} \\
\hline $0(n=261)$ & $12.0(5.4)$ & $15.8(6.4)$ & $0.81(0.17,0.93)$ & $-3.7(3.6)$ & $<0.001$ \\
\hline$\geq 1(n=239)$ & $12.1(4.0)$ & $16.5(4.5)$ & $0.61(-0.18,0.84)$ & $-4.4(3.4)$ & $<0.001$ \\
\hline \multicolumn{6}{|l|}{ Diagnosis } \\
\hline \multicolumn{6}{|l|}{ Left } \\
\hline POAG, PACG, and PXG $(n=390)$ & $11.7(4.3)$ & $16.0(4.9)$ & $0.69(-0.16,0.88)$ & $-4.3(3.2)$ & $<0.001$ \\
\hline Other $(n=110)^{\dagger}$ & $13.5(4.8)$ & $17.6(5.5)$ & $0.75(-0.8,91)$ & $-4.1(3.3)$ & $<0.001$ \\
\hline \multicolumn{6}{|l|}{ Right } \\
\hline POAG, PACG, and PXG $(n=399)$ & $11.7(4.7)$ & $15.8(5.5)$ & $0.74(-0.03,0.90)$ & $-4.1(3.5)$ & $<0.001$ \\
\hline Other $(n=101)^{\dagger}$ & $13.3(4.7)$ & $17.5(5.7)$ & $0.74(-0.5,90)$ & $-4.1(3.6)$ & $<0.001$ \\
\hline
\end{tabular}

Paired $t$ tests used to investigate differences except where indicated by ${ }^{\dagger}$ where Wilcoxon signed-rank tests were used. ICC, intraclass correlation coefficient; GAT, Goldmann applanation tonometry; IOP, intraocular pressure; CCT, central corneal thickness; SD, standard deviation. 
Fig. 1. IOP correlation between Icare and GAT for both eyes $(n=1,000)$. Solid line $=$ regression line, dotted lines $=95 \%$ confidence interval. IOP, intraocular pressure; GAT, Goldmann applanation tonometry.

Fig. 2. Bland-Altman diagram with difference in IOP ( $y$-axis), IOP means ( $x$-axis), between Icare and GAT measurements $(n=1,000)$. Solid line $=$ mean, dashed lines $=$ mean \pm SD. IOP, intraocular pressure; GAT, Goldmann applanation tonometry; SD, standard deviation.
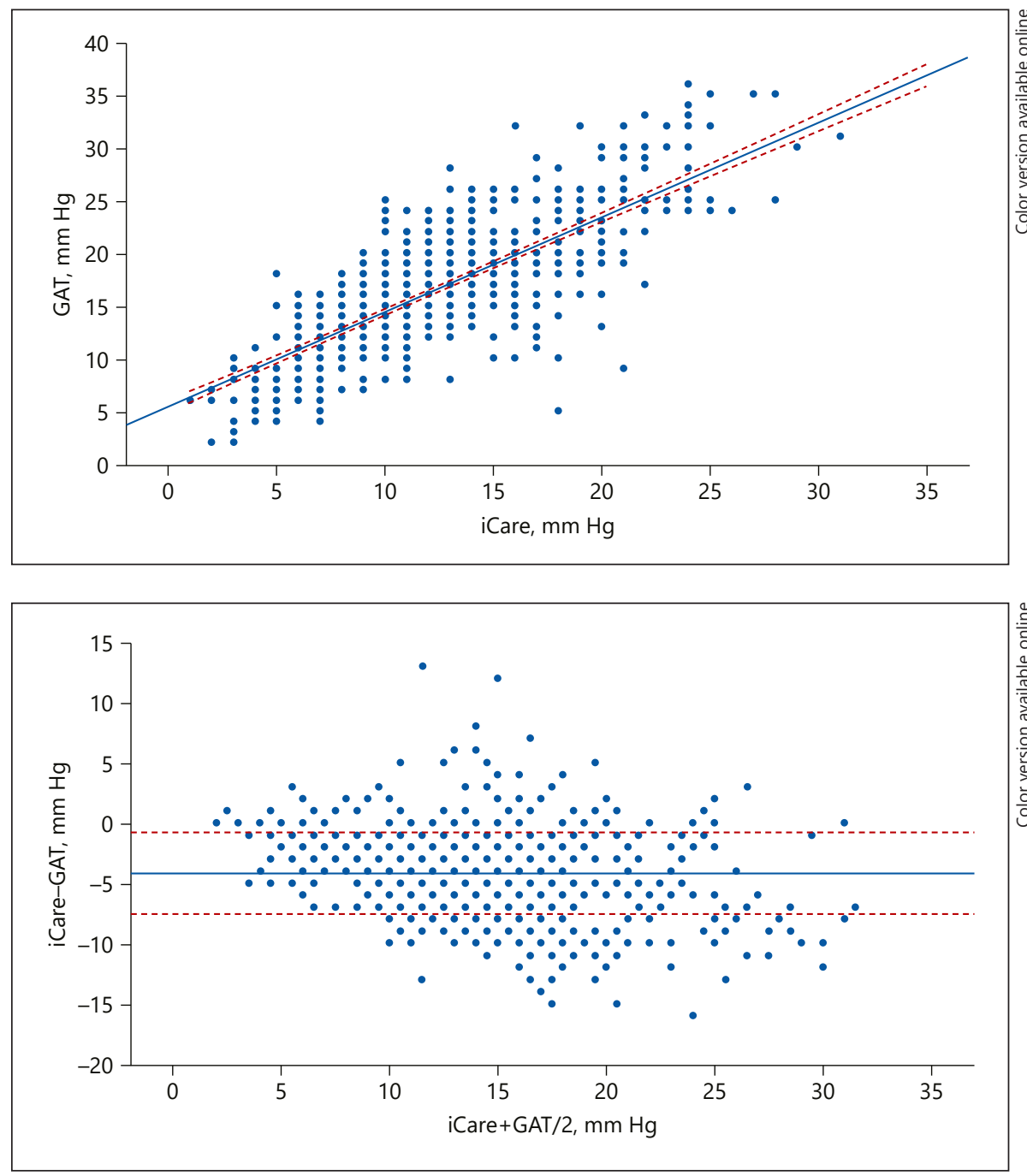

(80.1\%) eyes within 1 standard $1 \mathrm{SD}( \pm 3.4 \mathrm{~mm} \mathrm{Hg})$ of the mean difference $(-4 \mathrm{~mm} \mathrm{Hg})$ between Icare and GAT.

\section{Discussion/Conclusion}

As few studies have compared the Icare ic100 tonometer against GAT, we set out to address this gap by comparing IOP measurements obtained by this version of the Icare tonometer with the gold standard, GAT. We investigated IOP measured by Icare ic100 and GAT in 1,000 eyes, making this the largest study up-to-date comparing the 2 devices.

The Icare tonometer is marketed as a reliable and accurate device for measuring IOP using a unique mecha- nism to obtain its measurements. There are 5 different Icare tonometers on the market: the Icare TAOi, Icare Pro, Icare ic100, Icare ic200, and the Icare Home; however, each tonometer has its own characteristics and IOP value tendency.

Our study found the Icare ic100 consistently undermeasures IOP compared to GAT, with a mean difference of $4.2 \mathrm{~mm} \mathrm{Hg}$ (SD 4.3) between the Icare and GAT for all 1,000 eyes. This mean difference in IOP is considerably larger than the mean difference of $0.44 \mathrm{~mm} \mathrm{Hg}$ (SD 4.4) reported by Wong et al. [10] in their study of 74 eyes. It is also larger than the mean difference of $-2.5 \mathrm{~mm} \mathrm{Hg}$ (SD 2.8) reported by Nakakura [11] in a small study of 45 eyes, although it is equivalent to the -4.2 (SD 3.0) reported by Nakakura et al. [12] in a subsequent larger study of 106 eyes. 
Within the subgroups, the mean difference ranged between -5 and -3 . Exceptions to this were evident in patients with IOP ranges that are 12 and below, where the mean difference ranged from -1 to -2 and IOPs that are 22 and above, where the mean difference is -6 to -7 . Our findings suggest that the Icare underestimated the IOP measurement more so at the higher IOP ranges (i.e., 1321 and $\geq 22$ ). The largest mean differences in IOP measurement were in the $\geq 22$ IOP range in right eyes $(-6.8$ [SD 4.3]) and the $\geq 22$ IOP range in all eyes $(-6.8$ [SD 4.0]). This finding was similar to Wong et al. [10] who reported the largest mean differences in IOP measurement were among eyes with ocular hypertension. Similarly, in a study of 672 eyes using an earlier version of Icare (TA01i) Gao et al. [15] reported that the only subgroup with a statistically significant difference in IOP were eyes that had IOPs $\geq 23$.

Larger mean differences in IOP measurement were also observed in the $\geq 1$ medications group in each eye compared with the zero medications group in each eye. This may be explained by the $\geq 1$ medications group tending to have worse glaucoma, resulting in IOP in the higher ranges.

There was a good correlation between the IOP measurement by the Icare ic100 and IOP measurement by GAT, with an overall ICC of 0.73 between IOP measured by the 2 devices. This is similar to the ICC of 0.77 reported by Wong et al. [10]. Our overall ICC is higher than that reported by Nakakura et al. [12] who determined an ICC of 0.53 between Icare ic 100 and GAT. It is to be noted that the ICCs varied considerably within the subgroups, with ICCs in the IOP ranges of 13-21 (ICCs 0.32 to 0.34 ) and $\geq 22$ (ICCs 0.34 to 0.37 ), indicating poor correlation between IOP measurement by Icare ic100 and GAT. This reflects the wider range of IOP measurements by Icare ic100 compared with GAT within these subgroups.

The portability of the Icare tonometer device and its ease of use can benefit in the screening of patients and measuring IOP in situations where the usage of GAT is impractical. The accuracy of IOP measurement is important, especially in patients with severe glaucoma where further interventions may be appropriate in order to slow its progression and prevent further loss of vision [16]. Even $1 \mathrm{~mm} \mathrm{Hg}$ of IOP reduction had been shown to be associated with $10 \%$ reduction in glaucoma progression [17]. Surgeons need to be aware of this when Icare is used in their practice and should arguably carry out GAT in every glaucoma patient. Based on our findings, we feel more caution is needed when using the Icare ic 100 in high IOP ranges $(\geq 22)$ given the significant underestimation of IOP in that range compared to the GAT. The implications of underestimation of IOP can be significant in the management of glaucoma patients and this may include under-treatment of elevated IOPs leading to further visual loss.

There are some limitations to our study. First, the majority of the population we investigated were Caucasian, which limits the generalizability of the results to other ethnicities. While a single clinician obtained the GAT measurements, the Icare measurements were carried out by multiple technicians. In addition, in most cases, only 1 GAT measurement was recorded for practical reasons. Nonetheless, these were done by an experienced surgeon, who was also masked to the Icare measurements, and multiple measurements were taken in instances where there was any doubt of accuracy.

In conclusion, the Icare ic 100 consistently undermeasures IOP in comparison to the GAT in almost all subgroups. It is a user-friendly device that requires little training and does not require the use of topical anesthesia. There is still value in its use as a screening tool, and in the emergency department, however, caution may be needed in IOP ranges that are high $(\geq 22)$ due to Icare underestimating IOP. We strongly advise clinicians to carry out a routine GAT, especially in patients with advanced glaucoma.

\section{Acknowledgement}

The authors wish to thank Jennie Rossetto and the technical staff of the Tasmanian Eye Institute for providing practical support for us to conduct the study.

\section{Statement of Ethics}

The University of Tasmania Human Research Ethics Committee provided ethical approval for the research (reference: H18061). A waiver of consent for the research was granted, in accordance with section 2.3.10 of the Australian National Statement on Ethical Conduct in Human Research (2007, 2018 update), including the conditions that the research carried no more than low risk, and there was no known or likely reason for thinking that participants would not have consented if they had been asked. The research was performed according to the World Medical Association Declaration of Helsinki and conducted at 2 ophthalmology centers in Tasmania, Australia, between June and August 2019.

\section{Conflict of Interest Statement}

The authors have no conflicts of interest to declare. 


\section{Funding Sources}

The study was not externally funded.

\section{Author Contributions}

Arjun Gopal Subramaniam contributed to the interpretation of the data, drafting and revising the paper, and provided final approval for the version submitted. Penny Allen contributed to the data analysis and interpretation of the data, drafting of the paper, and provided final approval for the version submitted. Tze'Yo Toh contibuted to conception and design of the study, the acquisition of data and interpretation of the data, drafting of the paper, and provided final approval for the version submitted.

\section{References}

1 Stamper RL. A history of intraocular pressure and its measurement. Optom Vis Sci. 2011; 88(1):E16-28.

2 Salim S, Du H, Wan J. Comparison of intraocular pressure measurements and assessment of intraobserver and interobserver reproducibility with the portable ICare rebound tonometer and Goldmann applanation tonometer in glaucoma patients. J Glaucoma. 2013;22(4):325-9.

3 Hladíková E, Pluháček F, Marešová K. [Comparison of measurement of intraocular pressure by ICARE $\mathrm{PRO}^{\circ}$ tonometer and Goldman applanation tonometer]. Cesk Slov Oftalmol. 2014;70(3):90-3.

4 Rehnman JB, Martin L. Comparison of rebound and applanation tonometry in the management of patients treated for glaucoma or ocular hypertension. Ophthalmic Physiol Opt. 2008;28(4):382-6.

5 Motolko MA, Feldman F, Hyde M, Hudy D. Sources of variability in the results of applanation tonometry. Can J Ophthalmol. 1982; 17(3):93-5.

6 De Aplanacion CC. Rebound tonometry in a clinical setting. Comparison with applanation tonometry. Arch Soc Esp Oftalmol. 2007;82: 273-8.
7 Chen M, Zhang L, Xu J, Chen X, Gu Y, Ren Y, et al. Comparability of three intraocular pressure measurement: iCare pro rebound, noncontact and Goldmann applanation tonometry in different IOP group. BMC Ophthalmol. 2019 Dec;19(1): 1.

8 Pahlitzsch M, Brünner J, Gonnermann J, Maier AB, Torun N, Bertelmann E, et al. Comparison of ICare and IOPen vs Goldmann applanation tonometry according to international standards 8612 in glaucoma patients. Int J Ophthalmol. 2016;9(11):1624.

9 Pakrou N, Gray T, Mills R, Landers J, Craig J. Clinical comparison of the Icare tonometer and Goldmann applanation tonometry. J Glaucoma. 2008;17(1):43-7.

10 Wong B, Parikh D, Rosen L, Gorski M, Angelilli A, Shih C. Comparison of disposable Goldmann applanation tonometer, Icare iC100, and Tonopen XL to standards of care Goldmann nondisposable applanation tonometer for measuring intraocular pressure. J Glaucoma. 2018;27(12):1119-24.

11 Nakakura S. Icare ${ }^{\circledast}$ rebound tonometers: review of their characteristics and ease of use. Clin Ophthalmol. 2018;12:1245-53.
12 Nakakura S, Mori E, Fujio Y, Fujisawa Y, Matsuya K, Kobayashi Y, et al. Comparison of the intraocular pressure measured using the new rebound tonometer Icare $\mathrm{iC} 100$ and Icare TA01i or Goldmann applanation tonometer. J Glaucoma. 2019;28(2):172-7.

13 Brusini P, Salvetat ML, Zeppieri M, Tosoni C, Parisi L. Comparison of ICare tonometer with Goldmann applanation tonometer in glaucoma patients. J Glaucoma. 2006;15(3):213-7.

14 Phelps C, Phelps G. Measurement of intraocular pressure: a study of its reproducibility. Albrecht Von Graefes Arch Klin Exp Ophthalmol. 1976;198(1):39-43.

15 Gao F, Liu X, Zhao Q, Pan Y. Comparison of the iCare rebound tonometer and the Goldmann applanation tonometer. Exp Ther Med. 2017;13(5):1912-6.

16 Prum BE, Rosenberg LF, Gedde SJ, Mansberger SL, Stein JD, Moroi SE, et al. Primary open-angle glaucoma preferred practice Pattern $^{\circ}$ guidelines. Ophthalmology. 2016; 123(1):P41-111.

17 The AGIS Investigators. The Advanced Glaucoma Intervention Study (AGIS): 7. The relationship between control of intraocular pressure and visual field deterioration. Am J Ophthalmol. 2000;130:429-40. 